\title{
Unilateral hemorrhagic moyamoya disease and contralateral cavernous aneurysm in an Indian woman treated with stent-assisted coil technique: case report
}

\author{
Gautam Dutta ${ }^{*}$ D, Daljit Singh, Hukum Singh and Arvind Kumar Srivastava
}

\begin{abstract}
Background: Moyamoya disease (MMD) is a chronic, progressive occlusion of the arteries around the circle of Willis that leads to the development of characteristic collateral vessels seen on cerebral angiography. MMD is commonly accompanied by intracranial aneurysms; however, the current literature is sparse regarding the best available treatment options for aneurysms in patients with MMD. Long-term angiographic outcome is also not well established.

Case description: We report the safety and efficacy of stent-assisted coiling in an adult Indian woman of unilateral hemorrhagic MMD having a large asymptomatic cavernous segment aneurysm on the contralateral side. The patient had excellent angiographic occlusion without any recurrence/hemorrhage in the 5-year follow-up after the procedure.

Conclusion: MMD should be kept in mind in evaluating patients presenting with cerebrovascular accidents. The case strengthens the view that stent-assisted coil embolization of large saccular aneurysms around the circle of Willis is a viable minimally invasive therapeutic option in such unusual patients.
\end{abstract}

Keywords: Unilateral moyamoya disease, Aneurysm, Stent-assisted coiling, Cerebral hemorrhage

\section{Introduction}

Moyamoya disease (MMD) is a chronic cerebrovascular disorder of slow progressive occlusion or stenosis of terminal portion of the intracranial internal carotid artery (ICA) or proximal portion of the anterior cerebral artery (ACA) and/or middle cerebral artery (MCA). These abnormal collateral vessels exhibit a "puff of smoke" appearance (Moyamoya vessels) that are observed in the vicinity of these arterial lesions [1]. The Research Committee on Moyamoya disease of the Ministry of health and welfare of Japan (RCMJ) set diagnostic criteria considering only cases with bilateral lesions as definite MMD [2]. However, there are some instances of probable MMD which show unilateral involvement with angiographic findings similar to those of definite cases on the affected side. The suitable surgical management

\footnotetext{
* Correspondence: gautamblue@hotmail.com

Department of Neuro-Surgery, Govind Ballabh Pant Institute of Postgraduate Medical Education and Research (GIPMER), New Delhi 110002, India
}

option and long-term safety profile of the procedures performed in aneurysms associated with MMD is not well established. We herein report the case of a 55-yearold Indian woman with hemorrhagic stroke who exhibited a unilateral MMD with contralateral cavernous segment aneurysm managed with stent-assisted coiling and was followed up for 5 years. The current case proposes that stent-assisted coiling in saccular aneurysms located around the circle of Willis in such patients may be a safe and viable option with excellent long-term angiographic results.

\section{Case report}

A 55-year-old right-handed Indian woman without any history of head trauma or systemic medical disease and without any risk factor for arteriosclerosis was first seen in our department in the year 2012 after she experienced sudden headache, dizziness, nausea, and lethargy. She denied any alcohol use or cigarette smoking and was not 
on any medication. Her family history of any cerebrovascular disease was negative. On neurological examination, she was alert and oriented. The pupils were equal and reacted normally to light bilaterally. The fundi did not reveal any hemorrhage, exudate, or papilledema. She did not have any weakness of limbs and was having preserved sensory modalities. Cerebrovascular disease was suspected and computed tomography (CT) of the brain was obtained which revealed rounded hyperdense lesion in the right medial thalamus (Fig. 1a). Magnetic resonance imaging (MRI) of the brain demonstrated a small $(12 \times 8 \times 7 \mathrm{~mm})$ hemorrhagic lesion in the right medial thalamus which showed mixed intensity in all imaging sequences (Fig. 1b). Computed tomography angiography (CTA) and magnetic resonance angiography (MRA) of the brain was obtained which revealed marked stenosis of the supraclinoid segment of the right internal carotid artery (ICA) with non-visualization of both the ACA and their branches. The right MCA was very faintly visualized suggesting almost complete occlusion, left MCA and its branches, however, appeared relatively normal. The right ACA and MCA was appeared to be supplied by the posterior communicating artery. There was diffuse thinning of the P3 segment of the right posterior cerebral artery (PCA) with relatively normal left PCA.
The right vertebral artery was hypoplastic, and the left vertebral and basilar arteries appeared normal. Few prominent basilar and circle of Willis collaterals were apparent (Fig. 1c, d). Digital subtraction angiogram (DSA) indicated stenosis of the terminal portion of the right ICA and the proximal portions of the right MCA and ACA with Moyamoya vessels and aneurysm at the left ICA cavernous segment measuring $1.4 \times 1.2 \times 1 \mathrm{~cm}$ (Fig. 1e, f).

Symptomatic and supportive management was instituted. Stent-assisted coiling of the aneurysm was performed using four coils. Post-coiling angiogram confirmed complete occlusion of aneurysm sac and preserved distal flow. Postoperative course was uneventful, and the patient recovered well.

After discharge, the long-term outcome of the patient was ascertained through clinical visits in our outpatient department on a monthly basis for the initial 6 months of the procedure and then semiannually. CTA or magnetic resonance angiography (MRA) was done at 6 months follow-up and then yearly to look for contralateral progression, defined as any noticeable change on angiography on the contralateral site. DSA was obtained at 5 years follow-up (year, 2017) which revealed quiescent lesion without any contralateral progression

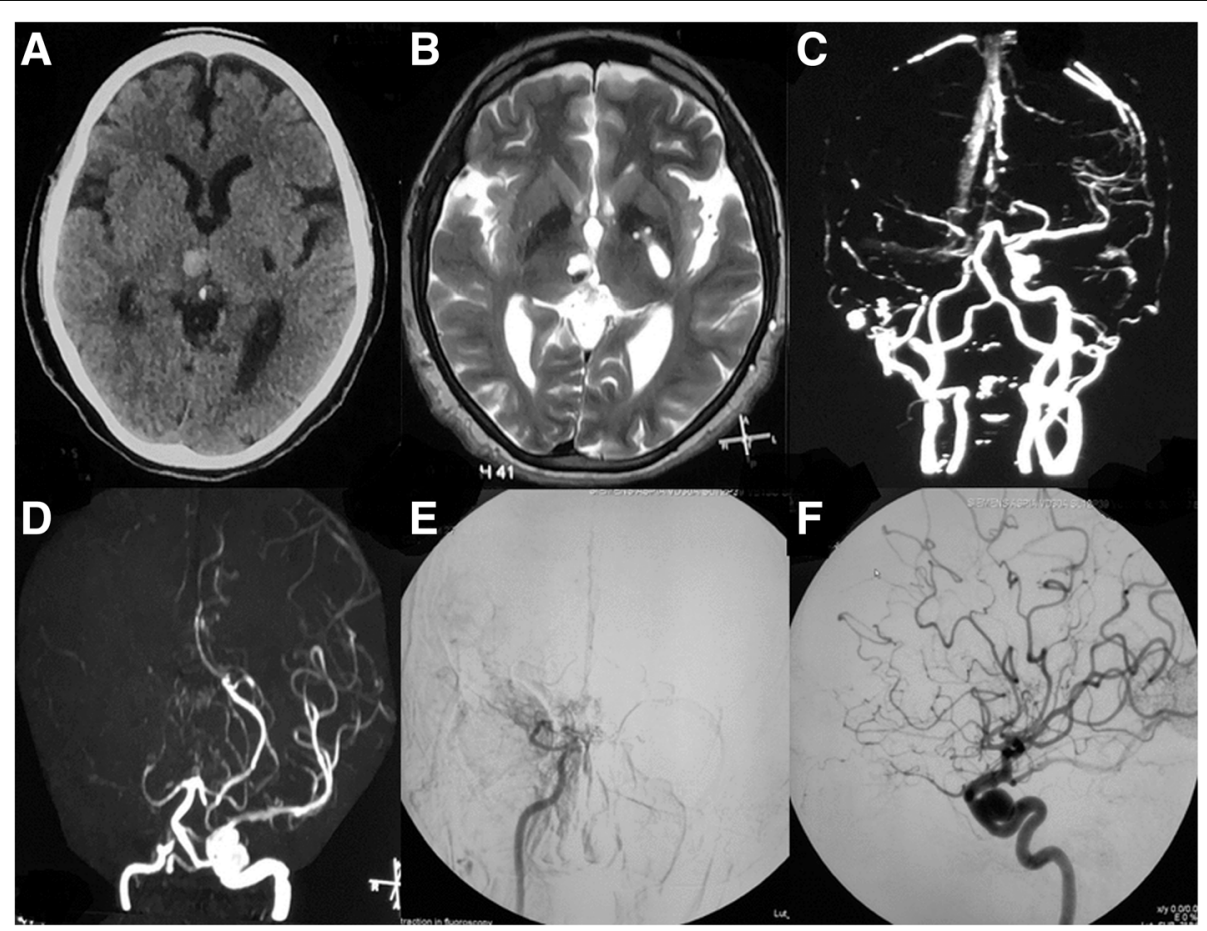

Fig. 1 a CT brain showing hyperdense lesion in the right medial thalamus. b T2-W MRI brain showing a $12 \times 8 \times 7$ mm hemorrhagic lesion in right medial thalamus. $\mathbf{c}$ CTA and $\mathbf{d}$ MRA brain showing marked stenosis of the supraclinoid segment of the right ICA with non-visualization of both the ACA and their branches with few prominent basilar and circle of Willis collaterals. e DSA image showing stenosis of the terminal portion of the right ICA and the proximal portions of the right MCA and ACA with Moyamoya vessels $\mathbf{f}$ DSA image showing a large aneurysm at the left ICA cavernous segment 
and good angiographic occlusion of the aneurysm sac (Fig. 2a, b).

\section{Discussion}

Whether unilateral lesion established by angiographic findings of MMD unilaterally and either equivocal or normal findings contralaterally is an early form of definite MMD remains controversial [3]. In a study of 64 patient with unilateral MMD followed up for 1-7 years, progression to bilateral disease was noted in 17 (27\%) patients, and such progression to bilateral disease within 5 years was more commonly seen in children with early onset of MMD [4]. Family history is sometimes associated with unilateral MMD [5]. Hence, it is thought that some of the unilateral cases are etiologically similar to definite MMD.

The fragile moyamoya vessels are more prone to rupture, and cerebral hemorrhage is the usual clinical presentation in adult patients with MMD, even in unilateral cases. Ikezaki et al. found that $58 \%$ of the adult unilateral MMD patients suffered from hemorrhagic stroke [5]. Not infrequently, MMD is seen to be associated with intracranial aneurysms, which is likely to be due to intrinsic pathology of the moyamoya vessels such as fragility and associated hemodynamic stress. These aneurysms are divided into three distinct subtypes: aneurysms at major artery (around the circle of Willis), distal peripheral arteries (such as the anterior or posterior choroidal artery), and those of moyamoya vessels (basal collaterals) [6]. A case of multiple aneurysms associated with unilateral MMD was reported by Kasamo et al., which resulted in subarachnoid hemorrhage [7].

The surgical management of these aneurysms poses a significant challenge owing to their deep location and frequent unfavorable morphology, and is complicated by hemodynamic fragility of the vessels [6]. Surgical revascularization is an option for peripheral aneurysms in the moyamoya vessels since hemodynamic stress reduction that ensues may result in disappearance of the aneurysms [8]. Recently, improving microvascular techniques and technology may enable more frequent endovascular treatment for these aneurysms [9, 10]. Aneurysms located at the circle of Willis are usually saccular aneurysms which are associated with high risk of bleeding, and revascularization is not a viable option in such cases as the procedure cannot make these aneurysms disappear. Clipping of such aneurysms has its own inherent risks. Dural handling and incisions may disturb the transdural collaterals [11], perforators and moyamoya vessels may cause hindrance [12], and placement of temporary clips at the parent vessel may cause irreversible hypoxic brain damage owing to an already compromised flow. Endovascular coil embolization could be a viable option in such cases. A study by Arita et al. [13] showed that conventional coil embolization is an efficient and safe treatment option for basilar tip aneurysms (BTA) associated with MMD.

Endovascular techniques using soft platinum coils are now considered to be alternatives for treating aneurysms in patients with aneurysms difficult to reach by craniotomy [14, 15]. Massoud et al. reported two BTA associated with MMD that was treated by endovascular embolization [16]. Two subsequent reports also documented excellent results $[17,18]$, and the current case also supports the safety and efficacy of coil embolization of aneurysms associated with MMD.

A literature search revealed no study showing efficacy of coil embolization in unilateral MMDs associated with cavernous aneurysms. Stent-assisted coiling is one modality which serves as a mechanical scaffold that promotes dense packing and prevents coil protrusion, and is safe with significantly lower recurrence, retreatment, and hemorrhage rates than coiling alone $[19,20]$. However, there is no study in literature to assess the efficacy

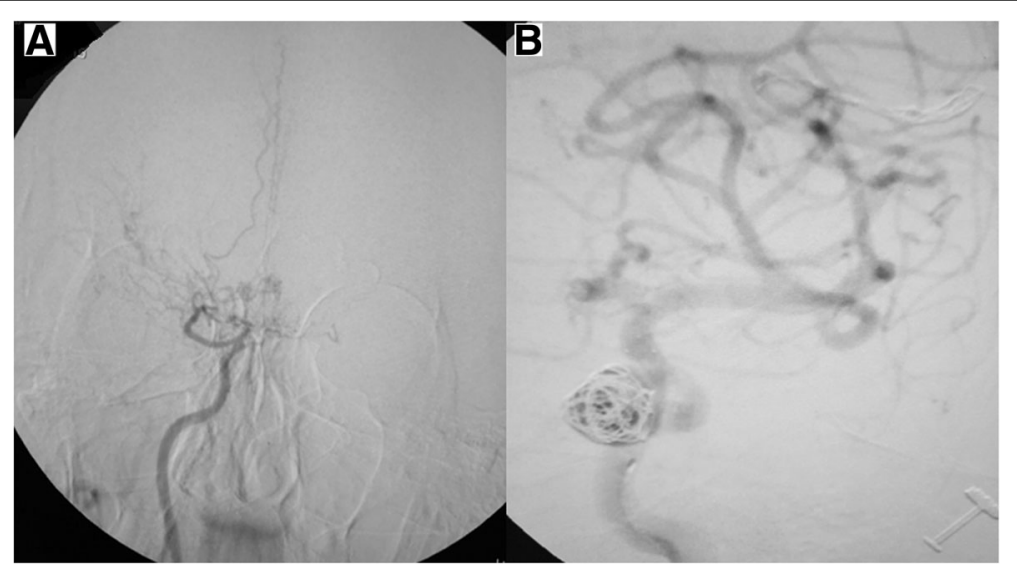

Fig. 2 a DSA image at 5 years follow-up showing persistent quiescent lesion and $\mathbf{b}$ without any contralateral progression and good angiographic occlusion of the aneurysm sac 
and long-term outcome of stent-assisted coiling in patients with unilateral MMD and cavernous segment aneurysms. Less data is available on endovascular management in MMD patients associated with aneurysm, and most of our interpretation is based on patchy case reports. Our patient underwent stent-assisted coiling which showed excellent angiographic occlusion even at 5 years follow-up suggesting this procedure may be a viable and safe option overcoming the risks associated with open approach in MMD patients harboring cavernous aneurysms.

Unilateral to bilateral progression in MMD is not well understood although some cases of unilateral MMD has been shown to be progressed to bilateral disease especially in children $[4,21,22]$. In a series of nine cases of unilateral MMD, no cases were seen to be progressed to bilateral involvement in another study [23]. Our case was followed up for 5 years and was not found to be progressing to involve the contralateral side. It is prudent to keep such patients in close observation to look for bilateral progression.

\section{Conclusion}

In conclusion, MMD should be suspected in patients presenting with cerebrovascular accidents. The present report strengthens the fact that some cases of unilateral moyamoya vessels may fall within the spectrum of MMD. Endovascular management using stent-assisted coil may be a safe and effective treatment modality for large or wide-neck aneurysms around the circle of Willis associated with MMD. However, the long-term safety of the procedure needs to be confirmed by more studies. We recommend endovascular management of such aneurysms in favorable cases as soon as they are diagnosed because of their tendency to rupture, followed by close observation to watch for progression to bilateral involvement.

\section{Abbreviations}

ACA: Anterior cerebral artery; CT: Computed tomography; DSA: Digital subtraction angiography; ICA: Internal carotid artery; MCA: Middle cerebral artery; MMD: Moyamoya disease; RCMJ: Research Committee on Moyamoya disease of the Ministry of health and welfare of Japan

\section{Acknowledgements}

Not applicable.

\section{Funding}

None.

\section{Availability of data and materials}

The data and material used during the current study is available from the corresponding author on reasonable request.

\section{Authors' contributions}

GD supervised, corrected, and proof read the manuscript. He was involved in the identification of the uniqueness of the case and giving care to the patient. DS was involved in examining and managing the patient. He also wrote the case summary of the patient. HS and AKS were involved in writing the rest of the manuscript. All authors read and approved the final manuscript.

\section{Ethics approval and consent to participate}

Not applicable (report of a single case). Written informed consent was however taken from the patient regarding possibility of publication of the case.

\section{Consent for publication}

The woman was briefed in detail about the uniqueness of case and its management, risk, benefits, and alternate mode of treatment.

Written informed consent was taken to report and publish the case.

\section{Competing interests}

The authors declare that they have no competing interests.

\section{Publisher's Note}

Springer Nature remains neutral with regard to jurisdictional claims in published maps and institutional affiliations.

Received: 15 June 2018 Accepted: 3 December 2018

Published online: 17 December 2018

\section{References}

1. Research Committee on the Pathology and Treatment of Spontaneous Occlusion of the Circle of Willis. Health Labour Sciences Research Grant for Research on Measures for Intractable Diseases: Guidelines for diagnosis and treatment of moyamoya disease (spontaneous occlusion of the circle of Willis). Neurol Med Chir (Tokyo). 2012;52:245-66.

2. Fukuji M. Guidelines for the diagnosis and treatment of spontaneous occlusion of the circle of Willis ('moyamoya' disease). Research committee on spontaneous occlusion of the circle of Willis (Moyamoya disease) of the Ministry of Health and Welfare, Japan. Clin Neurol Neurosurg. 1997;99(Suppl 2):S238-40.

3. Kelly ME, Bell-Stephens TE, Marks MP, Do HM, Steinberg GK. Progression of unilateral moyamoya disease: a clinical series. Cerebrovasc Dis. 2006; 22:109-15.

4. Kawano T, Fukui M, Hashimoto N, Yonekawa Y. Follow-up study of patients with "unilateral" moyamoya disease. Neurol Med Chir (Tokyo). 1994:34:744-7.

5. Ikezaki K, Inamura T, Kawano T, Fukui M. Clinical features of probable moyamoya disease in Japan. Clin Neurol Neurosurg. 1997;99:S173-7.

6. Kawaguchi S, Sakaki T, Morimoto T, Kakizaki T, Kamada K. Characteristics of intracranial aneurysms associated with moyamoya disease. A review of 111 cases. Acta Neurochir. 1996:138:1287-94.

7. Kasamo S, Asakura T, Yamamoto Y, Kobayashi E. Unilateral moyamoya disease associated with multiple aneurysms. A case report and review of the literature. Neurol Med Chir (Tokyo). 1984;24:30-4.

8. Kuroda S, Houkin K, Kamiyama H, Abe H. Effects of surgical revascularization on peripheral artery aneurysms in moyamoya disease: report of three cases. Neurosurgery. 2001:49:463-8

9. Harreld JH, Zomorodi AR. Embolization of an unruptured distal lenticulostriate aneurysm associated with moyamoya disease. AJNR Am J Neuroradiol. 2011;32:E42-3.

10. Kim SH, Kwon OK, Jung $\mathrm{CK}$, Kang $\mathrm{HS}$, Oh $\mathrm{CW}$, Han $\mathrm{MH}$, et al. Endovascular treatment of ruptured aneurysms or pseudoaneurysms on the collateral vessels in patients with moyamoya disease. Neurosurgery. 2009;65:1000-4.

11. Bhattacharjee AK, Tamaki N, Minami H, et al. Moyamoya disease associated with basilar tip aneurysm. J Clin Neurosci. 1999;6:268-71.

12. Nagamine Y, Takahashi S, Sonobe M. Multiple intracranial aneurysms associated with moyamoya disease. Case report. J Neurosurg. 1981;54: 673-6.

13. Arita K, Kurisu K, Ohba S, et al. Endovascular treatment of basilar tip aneurysms associated with moyamoya disease. Neuroradiology. 2003; 45:441-4

14. Guglielmi G, Viñuela F, Duckwiler G, et al. Endovascular treatment of posterior circulation aneurysms by electrothrombosis using electrically detachable coils. J Neurosurg. 1992;77:515-24.

15. Eskridge $\mathrm{JM}$, Song JK. Endovascular embolization of 150 basilar tip aneurysms with Guglielmi detachable coils: results of the Food and Drug Administration multicenter clinical trial. J Neurosurg. 1998:89:81-6.

16. Massoud TF, Guglielmi G, Viñuela F, et al. Saccular aneurysms in moyamoya disease: endovascular treatment using electrically detachable coils. Surg Neurol. 1994;41:462-7. 
17. Irie K, Kawanishi M, Nagao S. Endovascular treatment of basilar tip aneurysm associated with moyamoya disease_case report. Neurol Med Chir. 2000;40:515-8.

18. Kagawa K, Ezura M, Shirane R. Intraaneurysmal embolization of an unruptured basilar tip aneurysm associated with moyamoya disease. J Clin Neurosci. 2001; 8:462-4.

19. Chen Y, Dai D, Fang Y, Yang P, Huang Q, Zhao W, et al. Endovascular treatment of ruptured large or wide-neck basilar tip aneurysms associated with moyamoya disease using the stent-assisted coil technique. J Stroke Cerebrovasc Dis. 2015;24(10):2229-35.

20. Piotin M, Blanc R, Spelle L, et al. Stent-assisted coiling of intracranial aneurysms: clinical and angiographic results in 216 consecutive aneurysms. Stroke. 2010;41:110-5.

21. Kagawa R, Okada Y, Moritake K, Takamura M. Magnetic resonance angiography demonstrating adult moyamoya disease progressing from unilateral to bilateral involvement-case report. Neurol Med Chir. 2004;44:183-6.

22. Matsushima T, Inoue T, Natori Y, Fujii K, Fukui M, Hasuo K, et al. Children with unilateral occlusion or stenosis of the ICA associated with surrounding moyamoya vessels- "unilateral" moyamoya disease. Acta Neurochir. 1994; 131:196-202.

23. Hayashi K, Suyama K, Nagata I. Clinical features of unilateral moyamoya disease. Neurol Med Chir. 2010:50(5):378-85.

\section{Submit your manuscript to a SpringerOpen ${ }^{\circ}$ journal and benefit from:}

- Convenient online submission

- Rigorous peer review

- Open access: articles freely available online

- High visibility within the field

Retaining the copyright to your article

Submit your next manuscript at $\boldsymbol{\nabla}$ springeropen.com 